\title{
Measurement Distortion Analysis of Repetitive and Isolated Track Geometry Irregularities
}

\author{
Csaba Ágh $h^{1,2 *}$ \\ ${ }^{1}$ Doctoral School of Multidisciplinary Engineering Sciences, Széchenyi István University, Egyetem tér 1., H-9026 Győr, Hungary \\ 2 MÁV Central Rail and Track Inspection Ltd., Péceli u. 2., H-1097 Budapest, Hungary \\ * Corresponding author, e-mail: agh.csaba@sze.hu
}

Received: 05 December 2020, Accepted: 08 March 2021, Published online: 31 March 2021

\begin{abstract}
Track geometry measurements are essential for day-to-day activities of railway maintenance and play an important role in vehicletrack simulations. The generally applied forms of longitudinal level and alignment recordings do not reflect the real shape of the track. Both the versine measurement method and the band-pass filters according to European regulation cause significant amplitude modification and pattern change. In addition, the distortion behavior of repetitive and isolated defects is fundamentally different. In this contribution, simulated measurements of various reference shapes, which represent repetitive and isolated track deformations, were investigated. Comprehensive functions for amplitude change and for other distortion factors were developed with analytical and numerical methods. For chord measurements, rules were found for zero points and distortion-free ranges. Regarding the standardized filters, a significant amplitude reduction of isolated defects was observed in all wavelength ranges. Since derailment and track degradation depend not only on the amplitude of the defect, also the derivatives of the original and filtered forms of reference shapes were investigated and, as a new approach, the defect features called 'hypothetical additional force', 'speed of hypothetical wheel lift-off', 'hypothetical deterioration impulse' and 'hypothetical deterioration energy' were introduced.
\end{abstract}

Keywords

track geometry, versine measurement, wavelength domain filter, amplitude distortion, deterioration energy

\section{Introduction}

The principal track geometric parameters, immediate action limits of which are regulated by the technical specifications for interoperability relating to the 'infrastructure' subsystem of the rail system in the European Union [1], are: alignment, longitudinal level, track gauge, cant, and track twist. In this contribution, alignment and longitudinal level are investigated because it is not a trivial task to reconstruct their real values from the recordings provided by track measurement cars.

In some countries of the world, for example in Japan [2], the versine method is used for track maintenance purposes by track recording cars without further treatment. Moreover, versine method plays an important role in pre-measurements of tamping. Such signals are heavily dependent on the features of the chord establishing them. Equations for calculating the versines in horizontal and vertical curves were derived and published in [3]. The known formulae for amplitude and phase distortion of chord system transfer function [4] can be clearly attributed to the amplitude change and phase shift of the measurement signal of spatially periodic track deformation shapes. However, in reality, the most dangerous track defects are not periodic but isolated. Amplitude change and longitudinal peak shift of an isolated shape are not trivial and are fundamentally different from the aforementioned formulae. Therefore, this contribution investigates the amplitude and phase change as well as the full mathematical representation of distorted signal via different reference shapes, which represent both repetitive and isolated track deformations.

In the European Union, a 'decoloring' of the versine signals is obligatory in order to eliminate the influence of the transfer function of the chord system. Although there are a number of methods for decoloring, for example the traditional way based on the inverse transfer function of versine system [5] or the newly developed restoration model based on linear equations [6], the handling of zero points or low values of transfer functions remain a challenge in the practice because of, inter alia, numerical instability problems, 
background of which is detailed in [7]. Therefore, this contribution investigates zero points of the transfer functions and creates formulae which describe their occurrences comprehensively.

The already decolored signal may not be used on its own. According to the European regulations, different wavelength ranges shall be considered [8] which requires filtering methods. This is also the case for inertial measurements, which should also be filtered for the standardized wavelength domains. The use of these standardized filters is problematic because, in the reality, vehicles react to the full wavelength range. As Haigermoser et al. highlighted in [9], more vehement vehicle reaction is expected in a situation where two separate wavelength domains show high values than in a situation where only one of them shows even higher values. In addition, filters modify the original pattern of the track shape, including its amplitude. Therefore, this contribution investigates the distortive effect of the standardized filters D1 and D2 from the point of view of amplitude and other features by the help of the aforementioned reference track shapes.

In the international track maintenance practice, the tolerance limits refer usually only to amplitude values. However, theoretical considerations and simulations show that shorter waves lead to much higher forces than longer ones with the same amplitude $[10,11]$. Therefore, the investigated features and their distortion are calculated as a function of wavelength or, more specifically: as a function of the length of the considered reference defect shape.

It can furthermore be noted that derailment risk, which is crucial for track maintenance, depends primarily on the wheel-rail forces and not on defect amplitudes [12]. Considering a modern approach, track geometry quality evaluation can be made based on vehicle response prediction which focuses on vehicle accelerations and wheel-rail forces considering track wavelengths [10]. Such studies, for example [13], often use track geometry recordings filtered for wavelength domain D1 as an input. Filtered track geometry measurements are also used for assessing track degradation, for example in [14].

Since wheel-rail forces are determined by the second-order derivative of track shape $[15,16]$, and other effects are determined by the first-order derivative of track shape, in this contribution, the distortive effects of the standardized filters D1 and D2 were investigated also from the point of view of the derivatives on the corrupted shape (filtered signal of the reference track defect). In addition to the pure vertical wheel-rail force changes, as a new approach, the 'lift-off speed', 'deterioration impulse' and 'deterioration energy' were introduced and calculated based on the derivatives of the unfiltered and filtered longitudinal level signals.

It should be noted, incidentally, that there are many alternative methods in the literature for evaluating alignment defects: for example the 'spline method' [17], which was developed for evaluation of end points of transition curves (clothoide, hyperbolic [18], cosine).

\section{Methods}

\subsection{Considered reference track defects}

In this paper, three reference track shapes with wavelength $\lambda$ and amplitude $\alpha$, which are illustrated via Fig. 1 and defined in the following subsections, were investigated.

\subsubsection{Shape A (cyclic waving)}

Most of scientific contributions in this field investigate this periodic sinusoidal type of track deformation because it is mathematically simple, and its Fourier series can be easily handled. However, in practice, such a cyclic track deformation is not common, although the phenomenon called 'cyclic top', mentioned as a track geometry defect type in [8] and in [19], is in connection with it. Formula of this shape (Fig. 1(a)) is

$$
p_{A}(x)=\alpha \sin \left(\frac{2 \pi}{\lambda} x\right) \text {. }
$$

\subsubsection{Shape B (isolated deformation)}

Track geometry defect shape type (Fig. 1(b)), which can be approximated as

$p_{B}(x)=\left\{\begin{array}{cl}0 & \\ \alpha \cos \left(\frac{2 \pi}{\lambda} x\right)-\alpha, & , \quad 0 \leq x \leq \lambda, \\ 0 & , \lambda<x\end{array}\right.$

(a)

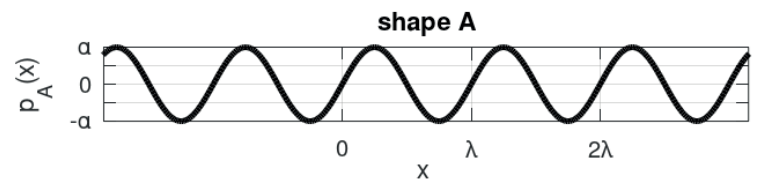

(b)

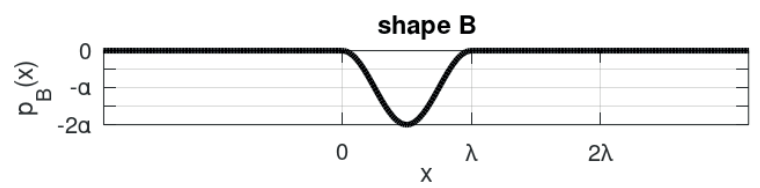

(c)

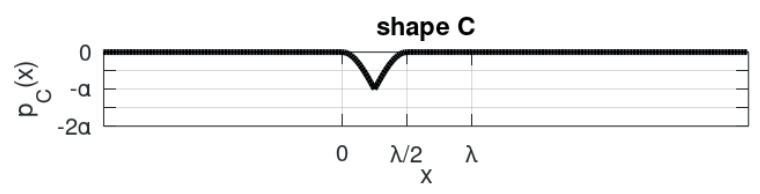

Fig. 1 Considered reference defect shapes 
is often the case in practice and called as 'local defect'. Since this function is non-periodic, it is more difficult to manage it with tools of mathematical analysis.

\subsubsection{Shape C (dipped rail joint)}

Dipped joints, also mentioned in the standard [8] create elbows in the rail. This was modeled by two parts of cosines. To make the elbow point differentiable infinite times, the edge was replaced by a very small half cosine shape (with a length of $\epsilon / 2$, all derivatives of which fit into the neighboring function parts' derivatives. Thus, definition of reference shape $\mathrm{C}$ (Fig. 1(c)) is:

(3)

$$
p_{C}(x)= \begin{cases}0, & x<0 \\ \alpha \cos \left(\frac{2 \pi}{\lambda} x\right)-\alpha, & 0 \leq x<\frac{\lambda}{4}-\frac{\epsilon}{4} \\ -\epsilon \alpha \cos \left[\frac{2 \pi}{\epsilon \lambda}\left(x-\frac{\lambda}{4}\right)\right]-\alpha, & \frac{\lambda}{4}-\frac{\epsilon \lambda}{4} \leq x<\frac{\lambda}{4}+\frac{\epsilon \lambda}{4} \\ -\alpha \cos \left(\frac{2 \pi}{\lambda} x\right)-\alpha, & \frac{\lambda}{4}+\frac{\epsilon}{4}<x \leq \frac{\lambda}{2} \\ 0, & \frac{\lambda}{2}<x\end{cases}
$$

where $\epsilon \rightarrow 0$.

\subsection{Considered measurement methods}

\subsubsection{Chord measurement}

Asymmetric chord measurement system, also called 'versine measurement', illustrated on Fig. 2(a) can be considered as a linear system, transfer function of which only depends on its chord length $(L)$ and chord division $(a$ and $b$ ). Complete characteristics of a linear system are described by its impulse response [20].

Hence, a chord measurement system realizes a convolution on the track shape $p(x)$ and its output $e(x)$ is calculated in the following way:

$$
e(x)=\int_{\xi=-\infty}^{+\infty} p(\xi) h(x-\xi) d \xi,
$$

where function $h(x)$ represents the unit impulse response of the system, illustrated in Fig. 2(c) as follows:

$$
h(x)=\left\{\begin{array}{ll}
-\frac{a}{L}, & x=-b \\
1, & x=0 \\
-\frac{b}{L}, & x=a \\
0, & x \neq-b, x \neq 0, x \neq a
\end{array} .\right.
$$
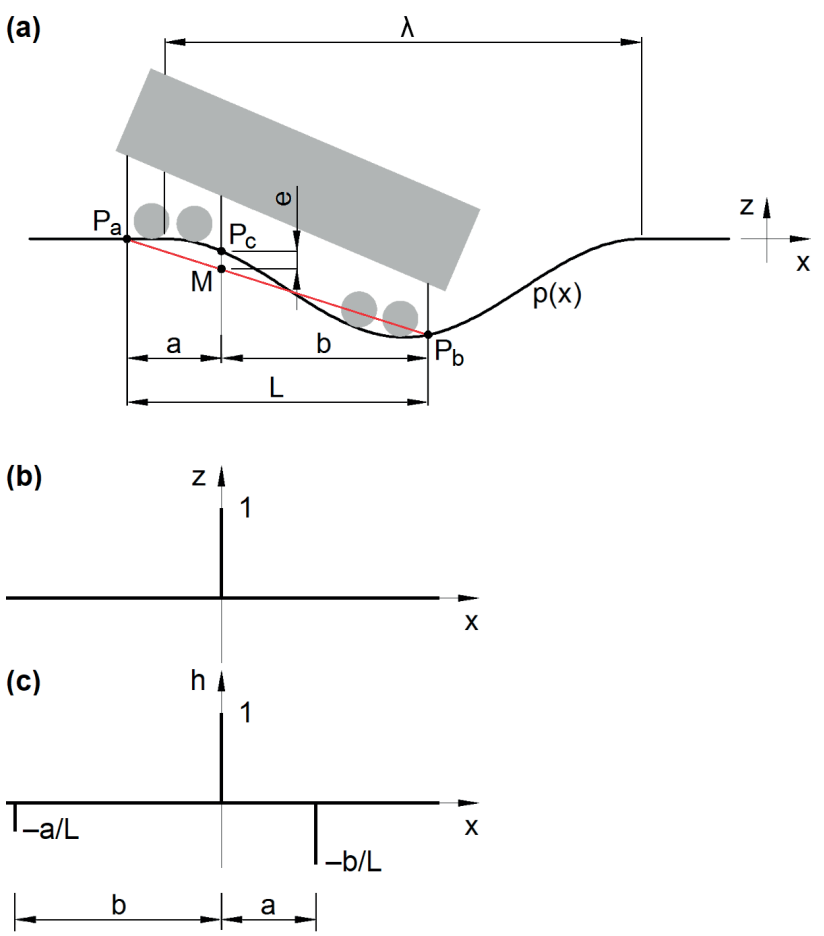

Fig. 2 Chord measurement system with measurement result $e$ (a); unit impulse on the track (b); unit impulse response $h$ of the system (c)

Since each sample of the output signal is equal to a sum of weighted inputs, to put Eq. (4) more simply, yields

$e(x)=p(x)-\frac{b}{L} p(x-a)-\frac{a}{L} p(x+b)$,

where

$L=a+b$ and $b \geq a>0$.

\section{Examination of Shape A}

Substituting Eq. (1) into Eq. (6), yields

$$
\begin{aligned}
& e_{A}(x)= \\
& \alpha\left[\sin \left(\frac{2 \pi x}{\lambda}\right)-\frac{b}{L} \sin \left(\frac{2 \pi(x-a)}{\lambda}\right)-\frac{a}{L} \sin \left(\frac{2 \pi(x+b)}{\lambda}\right)\right] .
\end{aligned}
$$

Applying trigonometric sum-to-product identity, Eq. (8) can be rewritten in following form:

$e_{A}(x)=\alpha\left[c_{A} \sin (\gamma)+d_{A} \cos (\gamma)\right]$

where

$$
\begin{aligned}
& \gamma=\frac{2 \pi}{\lambda} x \\
& c_{A}=1-\frac{a}{L} \cos \left(\frac{2 \pi}{\lambda} b\right)-\frac{b}{L} \cos \left(\frac{2 \pi}{\lambda} a\right) \\
& d_{A}=\frac{b}{L} \sin \left(\frac{2 \pi}{\lambda} a\right)-\frac{a}{L} \sin \left(\frac{2 \pi}{\lambda} b\right)
\end{aligned}
$$


As Eq. (9) represents phasors and it is known that sum of multiple phasors produces an another phasor. Based on the identity illustrated on Fig. 3 yields:

$e_{A}(x)=\alpha \sqrt{c_{A}^{2}+d_{A}^{2}} \sin \left(\gamma+\arctan \frac{d_{A}}{c_{A}}\right)$.

Some examples are presented via Fig. 4. In Fig. 4(a), the actual combination of wavelength, chord length and chord division leads to an amplitude magnification. By contrast, Fig. 4(b) represents an amplitude reduction and Fig. 4(c) shows total amplitude destruction. Comprehensive diagram of amplitude modification and phase modification factors is given in section 'Results'.

\section{Examination of Shape B}

Substituting Eq. (2) into Eq. (6) is more complicated. It is necessary to distinguish the following seven cases (Table 1), depending on the actual position of the leading, central, and trailing chord points, considering the tree parts of the track function .

If all three chord points are on the undeformed part of the track, the measurement result remains zero.

$e_{I}(x) \equiv 0$

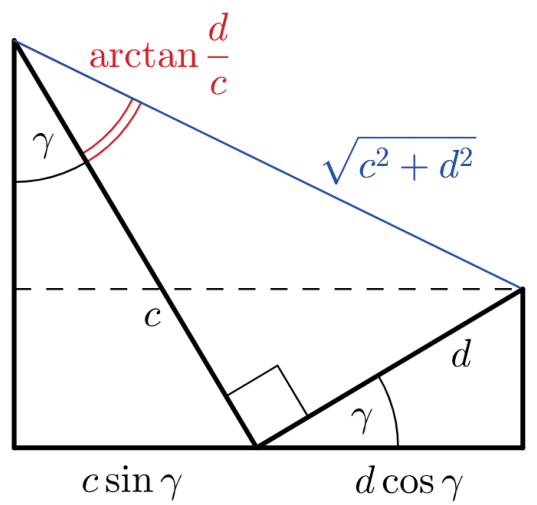

Fig. 3 Explanation of transformation of Eq. (9) into Eq. (13)

(a)

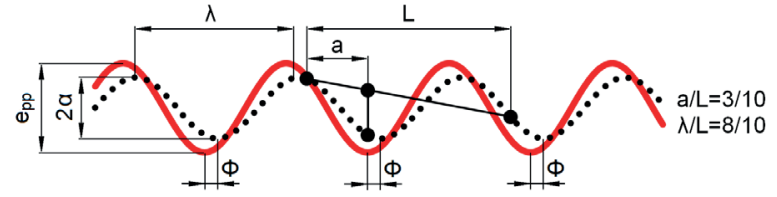

(b)

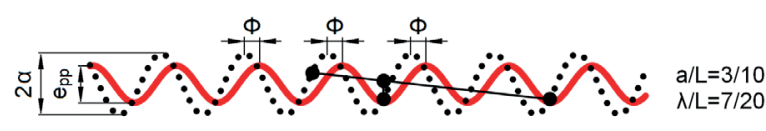

(c)

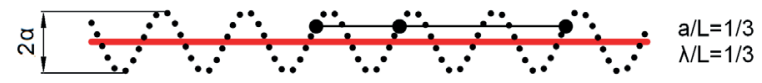

Fig. 4 Amplitude magnification (a); amplitude reduction (b) and amplitude destruction (c) of measurement (red) due to various asymmetrical chords (black continuous) over shape A (black dotted)
Table 1 Partitions of chord measurement graph in case of track shape B

\begin{tabular}{lcccc}
$\begin{array}{l}\text { segment } \\
\text { name }\end{array}$ & $\begin{array}{c}\text { position } \\
\text { of } P_{a}\end{array}$ & $\begin{array}{c}\text { position } \\
\text { of } P_{c}\end{array}$ & $\begin{array}{c}\text { position } \\
\text { of } P_{b}\end{array}$ & illustration \\
\hline$e_{\mathrm{I}}$ & 0 & 0 & 0 & cosine \\
$e_{\mathrm{II}}$ & 0 & 0 & cosine \\
$e_{\mathrm{III}}$ & 0 & cosine & cosine \\
$e_{\mathrm{IV}}$ & cosine & cosine & 0 & 0 \\
$e_{\mathrm{V}}$ & cosine & cosine & 0 & 0
\end{tabular}

When the first chord point $P_{b}$ arrives at the cosine deformation, measurement result function can be written as

$e_{I I}(x)=-\frac{a}{L}\left[\alpha \cos \left(\frac{2 \pi}{\lambda} x+\frac{2 \pi}{\lambda} b\right)-\alpha\right]$,

which represents a cosine in the opposite direction with amplitude factor of $a / L$ and phase shift of $2 \pi b / \lambda$. Similarly, when trailing $P_{a}$ point leaves the cosine:

$e_{V I}(x)=-\frac{b}{L}\left[\alpha \cos \left(\frac{2 \pi}{\lambda} x-\frac{2 \pi}{\lambda} a\right)-\alpha\right]$.

If both $P_{b}$ and $P_{c}$ points are on the cosine,

$e_{I I I}(x)=\alpha \cos \left(\frac{2 \pi x}{\lambda}\right)-\alpha-\frac{a}{L}\left[\alpha \cos \left(\frac{2 \pi x}{\lambda}+\frac{2 \pi b}{\lambda}\right)-\alpha\right]$,

which can be rewritten as

$e_{I I I}(x)=\alpha\left[c_{I I I} \sin (\gamma)+d_{I I I} \cos (\gamma)-1+\frac{a}{L}\right]$,

where $\gamma$ is according to Eq. (10) and

$c_{I I I}=\frac{a}{L} \sin \left(\frac{2 \pi}{\lambda} b\right)$,

$d_{I I I}=1-\frac{a}{L} \cos \left(\frac{2 \pi}{\lambda} b\right)$.

Eq. (18) can be transformed, in the same manner as shown via Fig. 3, into a form which represents an opportunity to demonstrate the amplitude and phase distortion:

$e_{I I I}(x)=\alpha\left[\sqrt{c_{I I I}^{2}+d_{I I I}^{2}} \cos \left(\gamma-\arctan \frac{c_{I I I}}{d_{I I I}}\right)-1+\frac{a}{L}\right]$.

Similarly,

$e_{V}(x)=\alpha\left[\sqrt{c_{V}^{2}+d_{V}^{2}} \cos \left(\gamma-\arctan \frac{c_{V}}{d_{V}}\right)-1+\frac{b}{L}\right]$,

where 
$c_{V}=-\frac{b}{L} \sin \left(\frac{2 \pi}{\lambda} a\right)$,

$d_{V}=1-\frac{b}{L} \cos \left(\frac{2 \pi}{\lambda} a\right)$.

By calculation of $e_{\mathrm{IV}}$, analyzing the equation

$$
\begin{aligned}
e_{I V}(x)= & \alpha\left\{\cos \left(\frac{2 \pi}{\lambda} x\right)-1-\frac{b}{L}\left[\cos \left(\frac{2 \pi}{\lambda}(x-a)\right)-1\right]\right. \\
& \left.-\frac{a}{L}\left[\cos \left(\frac{2 \pi}{\lambda}(x+b)\right)-1\right]\right\},
\end{aligned}
$$

it can be recognized that the situation emerged is similar to shape A, however, cosine is shifted upwards by one amplitude. Therefore

$e_{I V}(x)=\alpha \sqrt{c_{A}^{2}+d_{A}^{2}} \cos \left(\gamma+\arctan \frac{d_{A}}{c_{A}}\right)$.

It is an interesting situation, when only $P_{c}$ experiences the cosine and the chord ends are constantly at level 0 . In this case $e_{\mathrm{VII}}$ reflects the uncorrupted shape, defined by Eq. (2), of the track:

$e_{V I I}(x)=\alpha \cos \left(\frac{2 \pi}{\lambda} x\right)-\alpha$.

Fig. 5 shows two examples of measurement results with indication of function partitions I-VII mentioned above with different colors. Fig. 5(b) illustrates a situation where the absolute maximum value of the base-to-peak evaluation is fully compliant with the real defect size. Comprehensive visualization of amplitude modification and peak shift factors are given in section 'Results'.

Examination of Shape $C$

As in the case of shape B, equations can be created for chord measurement result partitions of shape $C$ (Fig. 6). But, for space reasons, they are not written in this contribution. Instead, distortion factors were calculated only via numerical simulations. A comprehensive visualization of amplitude modification factors is given in section 'Results'.

\subsubsection{D1 and D2 filtered measurement according to standard}

In this case, it is assumed that longitudinal level and alignment data series were recorded by an inertial system or by a chord measurement system, results of which had been already decolored. According to European regulations [1], railways should consider D1 and D2 wavelength domains, band-pass filters of which are standardized.
The zero phase Butterworth filters for D1 and D2 used in this contribution (Fig. 7) fulfill the requirements of the tolerance tables given in Annex $\mathrm{C}$ of European standard EN 13848-1:2019 [8].

Sampling interval of 0.25 meter is common practice on the field of track geometry recording. Therefore, the considered spatial sampling frequency in this contribution is $4 \mathrm{~m}^{-1}$. Cut-off frequencies (damping of $-3 \mathrm{~dB}$ where signal
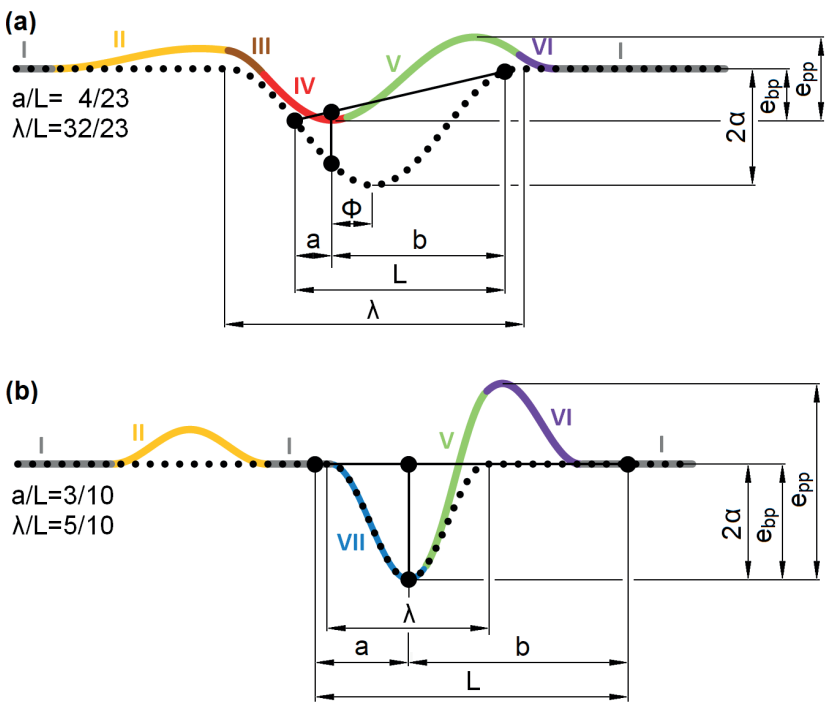

Fig. 5 Example measurement graphs $e_{\mathrm{I}-\mathrm{VII}}$ (colored) of asymmetrical chord systems (black continuous) in case of track deformation reference shape B (black dotted)

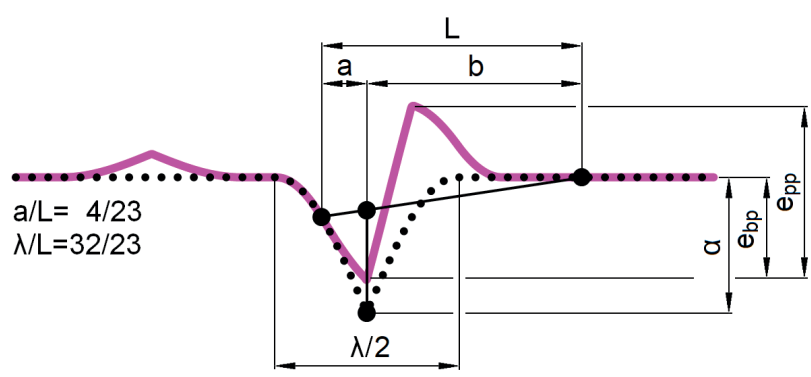

Fig. 6 Example measurement graph (colored) of asymmetrical chord system (black continuous) in case of track deformation shape $\mathrm{C}$ (black dotted)

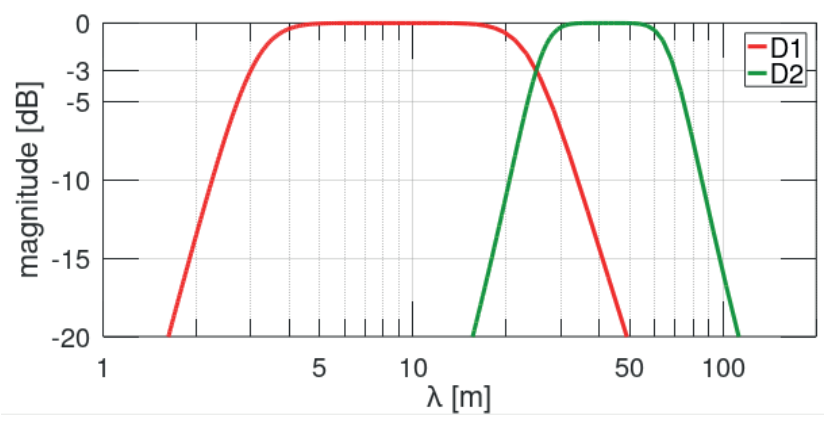

Fig. 7 Used filters for D1 and D2 wavelength domain 
power is halved and amplitude factor is $2^{-1 / 2}$ ) of D1 and D2 filters correspond to wavelengths $3 \ldots 25 \mathrm{~m}$ and $25 \ldots 70 \mathrm{~m}$, respectively. By simulations for all shapes $\mathrm{A}, \mathrm{B}, \mathrm{C}$ an interval of $[-5 \lambda, 5 \lambda]$ was considered because investigations showed that longer 'zero lines' before and after the track deformations B, C would not change the results. Fig. 8 shows examples of D1 filter results.

Comprehensive visualization of amplitude modification factors and the change of derivatives are given in section 'Results'.

\subsection{Calculated features}

In this section, traditional and new evaluation methods of aforementioned track geometry graphs are listed. They serve as a basis for distortion representation of chord measurement and standardized filtering. Numerical calculations were made by the Octave software.

\subsubsection{Base-to-peak values and peak-to-peak values}

The simplest evaluation method of track alignment and longitudinal level recordings is the so called 'base-to-peak' feature which considers positive or negative extrema relative to the zero line. European regulation [1] makes the use of this method compulsory for EU Member States regarding immediate action limits for isolated defects. Original 'base-to-peak' values for the reference shapes are easily readable in Fig. 1: for shape $\mathrm{A}$, the maximum/minimum deviation relative to the zero line is $\pm \alpha$, for shape $\mathrm{B}$ is $-2 \alpha$, for shape $\mathrm{C}$ is $-\alpha$. Other evaluation method of track geometry recordings is the so called 'peak-to-peak' value. It considers the absolute difference between the maximum positive and the maximum negative values of every two consecutive local extrema. For both shape A and shape B the deviation of consecutive maxima and minima relative

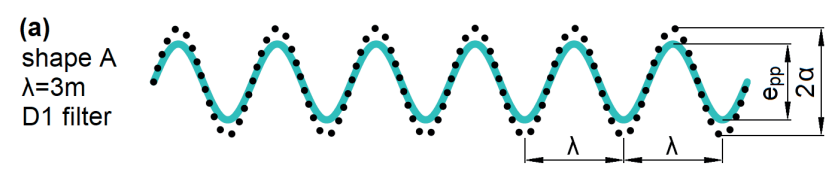

(b)

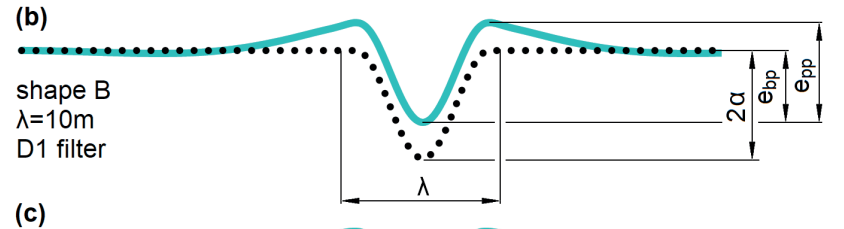

(c)

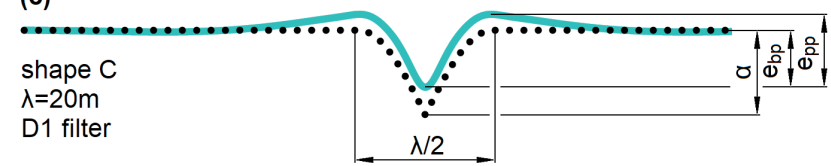

Fig. 8 Example measurement graphs of D1 filter (light blue) calculated on track reference track shapes A, B, C (black dotted) to each other is $2 \alpha$, and for shape $\mathrm{C}$ is $\alpha$ (Fig. 1). Using the notations of Fig. 4 and Fig. 8(a), the amplitude distortion factors for shape A are

$K_{A, b p}=K_{A, p p}=\frac{e_{b p}}{\alpha}=\frac{e_{p p}}{2 \alpha}=K_{A}$,

for isolated track defects with shape B, considering notations of Fig. 5 and Fig. 8(b), distortion factors are calculated as

$K_{B, b p}=\frac{e_{b p}}{2 \alpha}$

$K_{B, p p}=\frac{e_{p p}}{2 \alpha}$,

and for shape $\mathrm{C}$, considering notations of Fig. 6 and Fig. 8(c):

$$
\begin{aligned}
K_{C, b p} & =\frac{e_{b p}}{\alpha}, \\
K_{C, p p} & =\frac{e_{p p}}{\alpha} .
\end{aligned}
$$

The '40-m-moving-average' method, which is often used for filtering curves, is not considered in this contribution.

\subsubsection{Longitudinal peak shift (phase shift)}

In this contribution, the longitudinal peak shift (or phase shift) is the angle $\Phi$ in radians that the waveform has shifted from the certain reference point along the $x$ axis. Considering the notations of Fig. 4 and Fig. 5, phase shift for shapes A and B can be calculated based on the distance between the negative peak point of the track and the corresponding negative extremum in the transformed signal, relative to track wavelength $\lambda$ :

$\Phi=\frac{2 \pi}{\lambda} x\left(p_{\text {min }}\right)-x\left(e_{\text {min }}\right)$.

\subsubsection{Hypothetical additional force}

Track geometry measurements are used for assessing the safety risk of track geometry defects. From the point of view of derailment safety, components of wheel-rail force are a central issue to consider. As it was mentioned in section 'Introduction', vertical wheel-rail forces are determined by second-order derivatives of the function of the vertical track shape. It should be noted that this method cannot be applied in conjunction with alignment defects directly because wheel's lateral movement deviates from alignment of track. Assuming a rigid wheel following the vertical track shape $p$, with mass $m$, the additional vertical force $F_{z}$ acting between rail and track can be written as 
$F_{z}=\mathrm{m} a_{z}=\mathrm{m} \frac{\mathrm{d} v_{z}}{\mathrm{~d} t}=\mathrm{m} \frac{\mathrm{d}\left(\frac{\mathrm{d} z}{\mathrm{~d} t}\right)}{\mathrm{d} t}=\mathrm{m} \frac{\mathrm{d}^{2} z}{\mathrm{~d} t^{2}}=\mathrm{m} \frac{\mathrm{d}^{2} p}{\mathrm{~d} t^{2}}$,

where $v_{z}$ and $a_{z}$ are the vertical speed and vertical acceleration of the wheel, respectively. If $\mathrm{d} x$ is the length of the horizontal path travelled by the wheel during time $\mathrm{d} t$, furthermore $\mathrm{d} p$ is the length of the vertical path travelled by the wheel during time $\mathrm{d} t$, following equation can be found:

$\mathrm{d} t=\frac{\mathrm{d} x}{v_{x}}=\frac{\mathrm{d} p}{v_{z}}$,

where $v_{x}$ is the horizontal travelling speed. Since $v_{x}$ is considered to be constant along the track, Eq. (34) can be rewritten as

$$
F_{z}=\mathrm{m} v_{x}^{2} \frac{\mathrm{d}^{2} p}{\mathrm{~d} x^{2}} .
$$

Negative values of $F_{z}$ represent unburdening of track, relative to the original weight of wheel. During the numerical simulations, the second-order derivative was substituted in the following way:

$$
\frac{\mathrm{d}^{2} p}{\mathrm{~d} x^{2}} \approx p^{\prime \prime}=\frac{p(x-\Delta x)-2 p(x)+p(x+\Delta x)}{\Delta x^{2}},
$$

where $\Delta x$ is $0.25 \mathrm{~m}$.

\subsubsection{Speed of hypothetical wheel lift-off}

When vertical wheel-rail force $F_{z}$ equals to zero, wheel leaves the rail (Fig. 9). Such a situation also poses derailment risk and therefore it is worth investigating. Normally, vertical force acting on the wheel due to gravity is balanced by track in its entirety. In case of track deformation, additional negative vertical acceleration $a_{z}$ can reduce the weight of the wheel. At a theoretical critical lift-off situation, when the independent, rigid wheel just leaves the rail, following equation is fulfilled:

$\mathrm{mg}-\mathrm{m} a_{z}=0$,

where ' $\mathrm{g}$ ' is $-9.81 \mathrm{~m} \cdot \mathrm{s}^{-2}$. Considering Eqs. (34)-(36), the critical horizontal travelling speed $v_{x, l i f t}$ of the train is determined by the minimum value of second-order derivative of the track longitudinal level:

$$
v_{x, l i f t}=\sqrt{\frac{\mathrm{g}}{\min \left(\frac{\mathrm{d}^{2} p}{\mathrm{~d} x^{2}}\right)}} .
$$

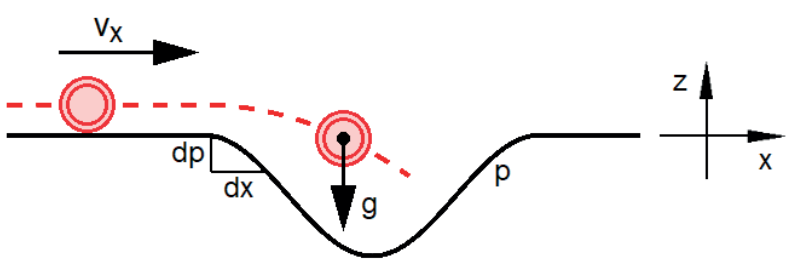

Fig. 9 Hypothetical 'wheel lift-off' over a longitudinal level defect

It should be noted that a lot of longitudinal level defects measured under loaded track condition originate from local deviation of foundation coefficient. In such a case there is no deformation in unloaded condition. In addition, springs of the railway vehicle improve safety against wheel lift-off, and this is not considered in this model. So, in the practice, lift-off cannot occur according to Eq. (39).

\subsubsection{Hypothetical deterioration impulse}

Assuming that gradual track deterioration is caused by additional vertical impulses $J$ acting on the wheel, and considering a simple wheel with constant speed as in the previous section, using the Newton-Leibniz formula and substituting Eq. (35):

$$
\begin{aligned}
J & =\int_{t_{1}}^{t_{2}} F_{z} \mathrm{~d} t=\mathrm{m} \int_{t_{1}}^{t_{2}} \frac{\mathrm{d}^{2} p}{\mathrm{~d} t^{2}} \mathrm{~d} t=\mathrm{m}\left\{\left[\frac{\mathrm{d} p}{\mathrm{~d} t}\right]_{t_{2}}-\left[\frac{\mathrm{d} p}{\mathrm{~d} t}\right]_{t_{1}}\right\} \\
& =\mathrm{m} v_{z}\left(t_{2}\right)-\mathrm{m} v_{z}\left(t_{1}\right)=\mathrm{m} v_{x}\left\{\left[\frac{\mathrm{d} p}{\mathrm{~d} x}\right]_{x_{2}}-\left[\frac{\mathrm{d} p}{\mathrm{~d} x}\right]_{x_{1}}\right\} .
\end{aligned}
$$

It can be concluded that the distortion impulse, in the terms of this paper, depends only from the change of the first-order derivative of the track shape which equals to the change of the vertical speed of wheel. Vertical speeds $v_{z}$ passing by reference shapes are the followings:

$$
\begin{aligned}
& v_{z, A}=\frac{\mathrm{d} p_{A}}{\mathrm{~d} x} \frac{\mathrm{d} x}{\mathrm{~d} t}=\frac{2 \pi \alpha v_{x}}{\lambda} \cos \frac{2 \pi}{\lambda} x, \\
& v_{z, B}=\frac{\mathrm{d} p_{B}}{\mathrm{~d} x} \frac{\mathrm{d} x}{\mathrm{~d} t}=-\frac{2 \pi \alpha v_{x}}{\lambda} \sin \frac{2 \pi}{\lambda} x, 0 \leq x \leq \lambda
\end{aligned}
$$

$$
v_{z, C}= \begin{cases}-\frac{2 \pi \alpha v_{x}}{\lambda} \sin \left(\frac{2 \pi}{\lambda} x\right), & 0 \leq x<\frac{\lambda}{4}-\frac{\epsilon}{4} \\ \frac{2 \pi \alpha v_{x}}{\lambda} \sin \left[\frac{2 \pi}{\epsilon \lambda}\left(x-\frac{\lambda}{4}\right)\right], & \frac{\lambda}{4}-\frac{\epsilon \lambda}{4} \leq x<\frac{\lambda}{4}+\frac{\epsilon \lambda}{4}, \\ \frac{2 \pi \alpha v_{x}}{\lambda} \sin \left(\frac{2 \pi}{\lambda} x\right), & \frac{\lambda}{4}+\frac{\epsilon}{4}<x \leq \frac{\lambda}{2}\end{cases}
$$

where $\epsilon \rightarrow 0$. 
During the numerical simulations, the first order derivative was substituted in the following way:

$$
\frac{\mathrm{d} p}{\mathrm{~d} x} \approx p^{\prime}=\frac{p(x)-p(x-\Delta x)}{\Delta x},
$$

where $\Delta x$ is $0.25 \mathrm{~m}$. An example is shown in Fig. 10 .

\subsubsection{Hypothetical deterioration energy}

Similar to the impulse, vertical kinetic energy change $\Delta E_{k}$ imparted to the wheel from the vehicle-track system while passing the track deformation can be calculated as:

$W=\Delta E_{k i n}=\int_{z_{1}}^{z_{2}} F_{z} \mathrm{~d} z=\int_{p_{1}}^{p_{2}} F_{z} \mathrm{~d} p$

Substituting Eq. (36) and assuming a constant travelling speed:

$W=\mathrm{m} v_{x}^{2} \int_{p_{1}}^{p_{2}} \frac{\mathrm{d}^{2} p}{\mathrm{~d} x^{2}} \mathrm{~d} p$.

Integration over vertical distance can be changed to the integration over horizontal distance in the following way:

$W=\mathrm{m} v_{x}^{2} \int_{p_{1}}^{p_{2}} \frac{\mathrm{d}^{2} p}{\mathrm{~d} x^{2}} \frac{\mathrm{d} p \mathrm{~d} x}{\mathrm{~d} x}=\mathrm{m} v_{x}^{2} \int_{x_{1}}^{x_{2}}\left(\frac{\mathrm{d}^{2} p}{\mathrm{~d} x^{2}} \frac{\mathrm{d} p}{\mathrm{~d} x}\right) \mathrm{d} x$.

Searching for an appropriate primitive function, it can be found based on the product rule of calculus that

$\frac{\mathrm{d}\left(\frac{\mathrm{d} p}{\mathrm{~d} x}\right)^{2}}{\mathrm{~d} x}=\frac{\mathrm{d}^{2} p}{\mathrm{~d} x^{2}} \frac{\mathrm{d} p}{\mathrm{~d} x}+\frac{\mathrm{d} p}{\mathrm{~d} x} \frac{\mathrm{d}^{2} p}{\mathrm{~d} x^{2}}=2 \frac{\mathrm{d}^{2} p}{\mathrm{~d} x^{2}} \frac{\mathrm{d} p}{\mathrm{~d} x}$.

Therefore, using the Newton-Leibniz formula, it yields

$$
W=\frac{\mathrm{m} v_{x}^{2}}{2}\left\{\left[\left(\frac{\mathrm{d} p}{\mathrm{~d} x}\right)^{2}\right]_{x_{2}}-\left[\left(\frac{\mathrm{d} p}{\mathrm{~d} x}\right)^{2}\right]_{x_{1}}\right\},
$$

which can be rewritten using Eq. (35) as

$$
W=\frac{\mathrm{m}}{2}\left[v_{z}^{2}\left(x_{2}\right)-v_{z}^{2}\left(x_{1}\right)\right] \text {. }
$$

It can be concluded that the distortion energy change, in terms of this contribution, depends on the change of the square of vertical speed of wheel which is determined by the first-order derivative of the track shape. During the computations, the first-order derivative was substituted in the aforementioned way.

As an example, Fig. 10 represents the zero-order, first-order and second-order derivatives (via original and D1, D2 filtered forms) of all three reference shapes with wavelength of 18 meters. Because neither filtering nor
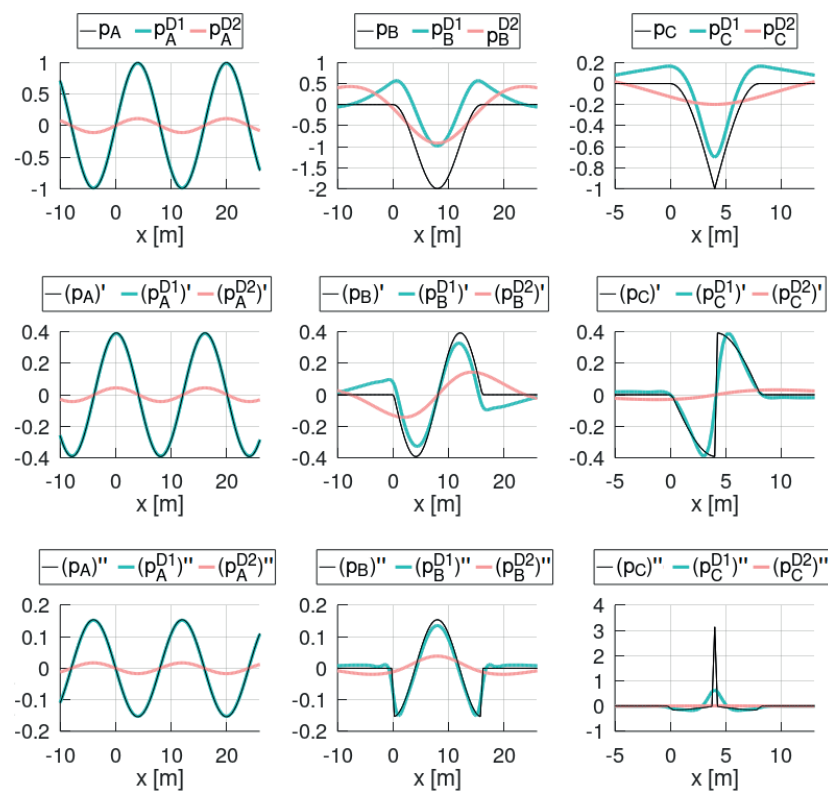

Fig. 10 Original and D1/D2 filtered form (first row) of reference shapes A, B, C with wavelength $\lambda=18.0 \mathrm{~m}$, amplitude $\alpha=1$, and their first-order (second row) and second-order (third row) derivatives with discretization step of $\Delta x=0.25 \mathrm{~m}$

differentiation is sensitive to the multiplication of the amplitude, vertical axes contain dimensionless numbers.

A comprehensive evaluation of derivatives (from the point of view of hypothetical additional force, speed of hypothetical wheel lift-off, hypothetical deterioration impulse and hypothetical deterioration energy) covering all wavelengths is presented in section 'Results'.

\section{Results}

\subsection{Distortion of base-to-peak and peak-to-peak values} Amplitude distortion factors were investigated according to the methods of Section 2.3.1.

\subsubsection{Amplitude distortion factor of chord method Shape A}

Comparing Eq. (1) with Eq. (13), it can be recognized that chord measurement transforms original sine into a sine with the same period but changed amplitude of

$K_{A}^{\text {chord }}=\sqrt{c_{A}^{2}+d_{A}^{2}}$.

Amplitude distortion function $K_{A}^{\text {chord }}$, which equals to the absolute value of the transfer function of the chord system [4], is only dependent on the chord measures and their ratio to the wavelength. Distortion values were plotted in two different manners: as a function of variables $\lambda / L, a / L$ (Fig. 11(a)) and as a function of variables $\mathrm{a} / \lambda, \mathrm{b} / \lambda$. (Fig. 11(b)). Both representations are comprehensive. 


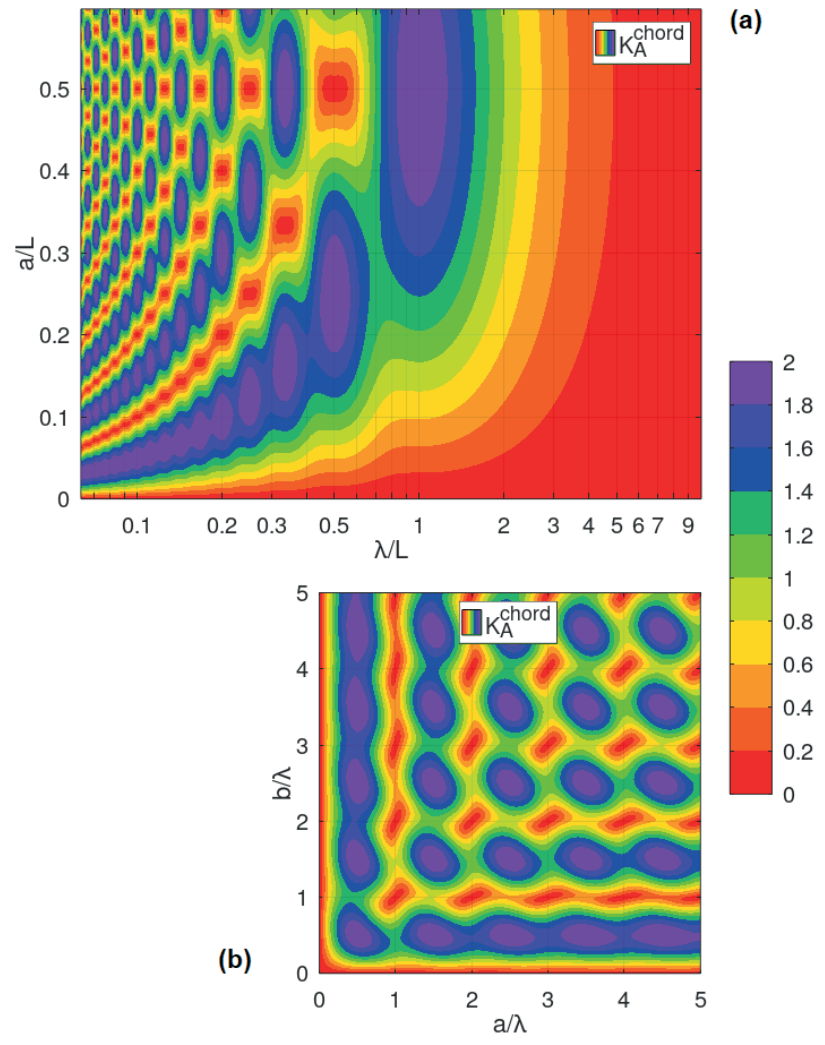

Fig. 11 Amplitude distortion factor of shape A due to chord measurement with wavelength $\lambda$, chord length $L$ and chord part lengths $a, b$ as a function of $\lambda / L$ and $a / L$ (a), furthermore as a function of $a / \lambda$ and $b / \lambda$ (b)

Under certain conditions, the value of the amplification factor is 0 . These zero points occur where the magnitude of the measurement result disappears (Fig. 4(c)). On the basis of Eq. (51), zero points of function $K_{A}^{\text {chord }}$ have been analyzed. Trivial zero points appear when chord part length $\mathrm{a}$ or $\mathrm{b}$ is 0 . Non-trivial zero points can be defined as followings. Function takes the value of 0 if both Eq. (52) and Eq. (53) are fulfilled:

$a=\zeta_{1} \lambda, \quad \zeta_{1} \in \mathbb{Z}^{+}$,

$b=\zeta_{2} \lambda, \zeta_{2} \in \mathbb{Z}^{+}$.

This means that all symmetric and asymmetric chord measurement systems have infinite number of zero points in the transfer function. Zero points occur where both chord parts $a$ and $b$ are positive integral multiples of the wavelength $\lambda$. This fact is illustrated via Fig. 11(b), where zero points occur in grid points.

Shape $B$ and $C$

Fig. 5 presents distorted function segments of shape B listed by Table 1 and Eqs. (14)-(27), with different colors.

Amplitude modification factors for shape B were visualized in Fig. 12(a) and Fig. 12(b). It can be stated that base-to-peak amplitude in the versine measurement of
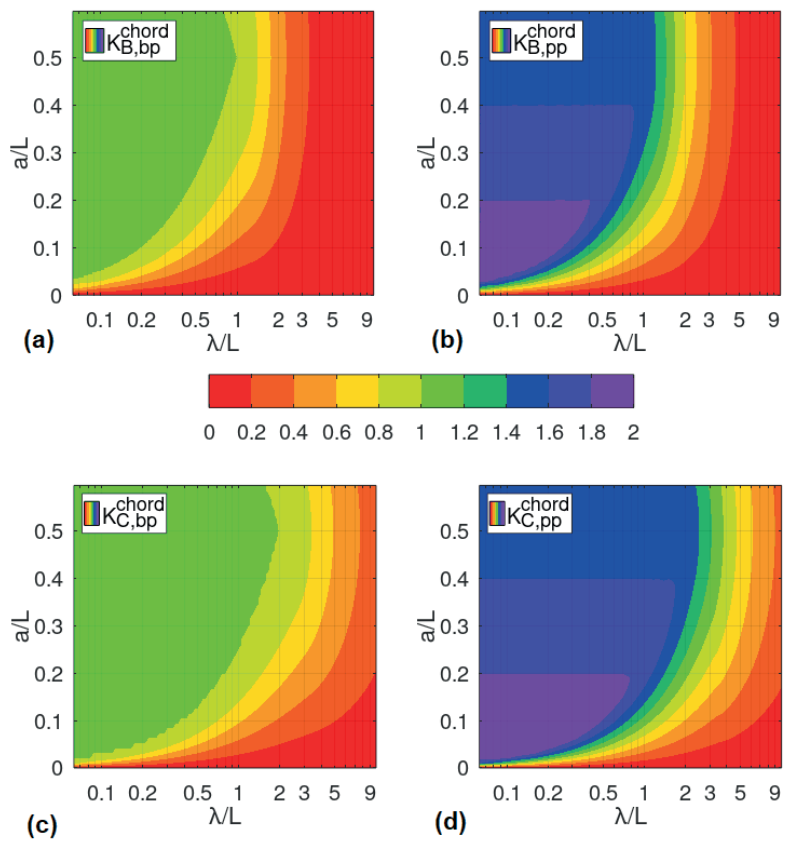

Fig. 12 Amplitude distortion factor of shapes B and $\mathrm{C}$ due to chord measurement with wavelength $\lambda$, chord length $L$ and chord part length $a$ in the cases of base-to-peak (bp) and peak-to-peak (pp) evaluation

shape $\mathrm{B}$ equals to the original amplitude (it means that $\left.K_{B, b p}^{c h o r d}=1\right)$ if inequation of $2 a \geq \lambda$ is fulfilled. This phenomenon was illustrated also via Fig. 5(b).

For shape $\mathrm{C}$, the distortion factors were visualized in Fig 12(c) and Fig. 12(d) and a similar statement can be made: base-to-peak amplitude in the versine measurement of shape $\mathrm{C}$ equals to the original amplitude (it means that $K_{C, b p}^{\text {chord }}=1$ ) if inequation of ' $2 a \geq \lambda / 2$ ' is fulfilled. Summarizingly, base-to-peak evaluation of isolated defect is free of distortion if the length of the shape is not longer than twice of the shorter chord part. In all other cases the amplitude factor $K_{B, b p}^{\text {chord }}=1$ or $K_{C, b p}^{\text {chord }}=1$ is less than 1 .

\subsubsection{Amplitude distortion factor of D1 and D2 filter}

Using the method written in Section 2.3.1, amplitude distortion factors were calculated on discretized 'filtered reference track shapes' illustrated via Fig. 8, using the filters detailed in Section 2.2.2.

The related results are shown in Fig. 13. It was found that for periodic sinusoidal track deformations (shape A) the filtered amplitudes correspond to the filter characteristics. However, for isolated defects (shapes B and C) the filtered amplitudes are always smaller, and the reduction is significant.

Following statements regarding maximum and minimum amplitudes apply to filtering for domain D1. The filtered base-to-peak amplitudes of shape B represent 77 $\%, 83 \%$ and $29 \%$ of the original amplitudes in case of 
(a)

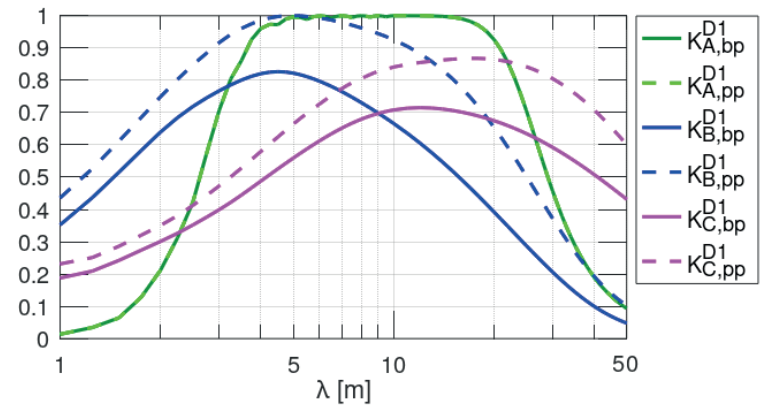

(b)

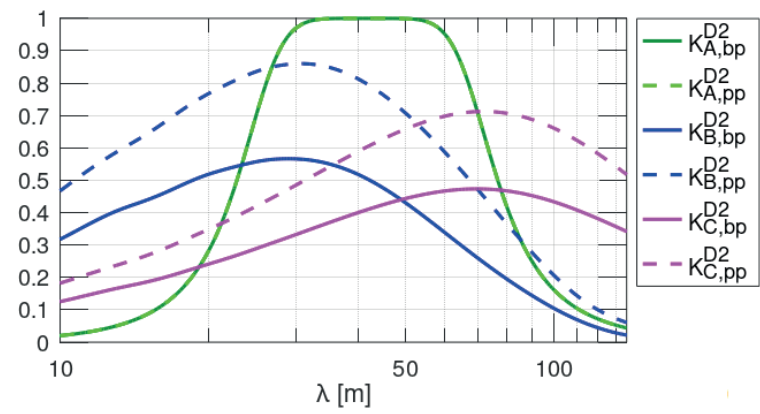

Fig. 13 Amplitude distortion factor of D1 (a) and D2 (b) filter on reference shapes A, B, C with wavelength $\lambda$ in the cases of base-to-peak (bp) and peak-to-peak (pp) evaluation

defect wavelengths $3 \mathrm{~m}, 4.5 \mathrm{~m}$ and $25 \mathrm{~m}$, respectively. The filtered peak-to-peak amplitudes of shape B represent $92 \%, 100 \%$ and $50 \%$ of the original amplitudes in case of defect wavelengths $3 \mathrm{~m}, 5 \mathrm{~m}$ and $25 \mathrm{~m}$, respectively. The filtered base-to-peak amplitudes of shape $\mathrm{C}$ represent $40 \%, 72 \%$ and $64 \%$ of the original amplitudes in case of defect wavelengths $3 \mathrm{~m}, 12 \mathrm{~m}$ and $25 \mathrm{~m}$, respectively. The filtered peak-to-peak amplitudes of shape $\mathrm{C}$ represent $47 \%, 87 \%$ and $84 \%$ of the original amplitudes in case of defect wavelengths $3 \mathrm{~m}, 17 \mathrm{~m}$ and $25 \mathrm{~m}$, respectively.

\subsection{Longitudinal peak shift (phase shift)}

Comparing Eq. (1) with Eq. (13), it can be recognized that chord measurement method transforms original sine into a sine with the same period but with phase shift of

$\Phi_{A}^{\text {chord }}=\arctan \left(d_{A} / c_{A}\right)$,

where $c_{A}$ and $d_{A}$ were defined via Eqs. (11)-(12).

According to method described in Section 2.3.2, values of $\Phi_{A}^{\text {chord }}$ calculated by the help of Eq. (54) and values of $\Phi_{B}^{\text {chord }}$ calculated by the help of a numerical simulation are shown by Fig. 14, considering Eq. (33).

In the case of chord measurement of shape $\mathrm{C}$, there is no longitudinal peak shift because negative peak of measurement graph is always at the negative peak of the track.

D1 and D2 filters are zero-phase filters, therefore they do not cause any phase shift.

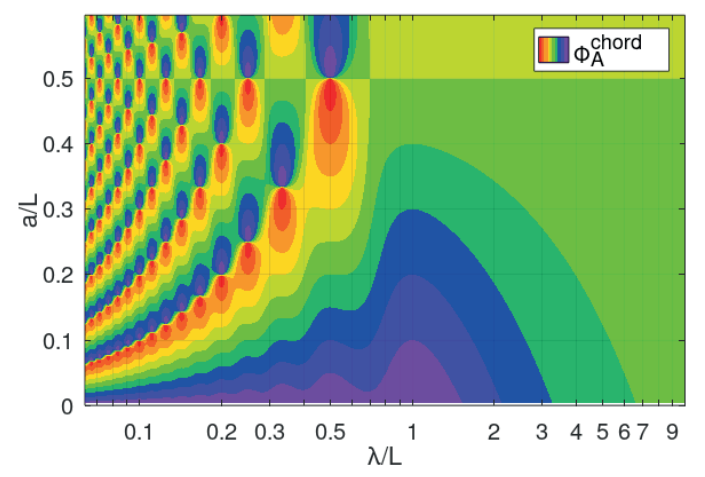

(a)

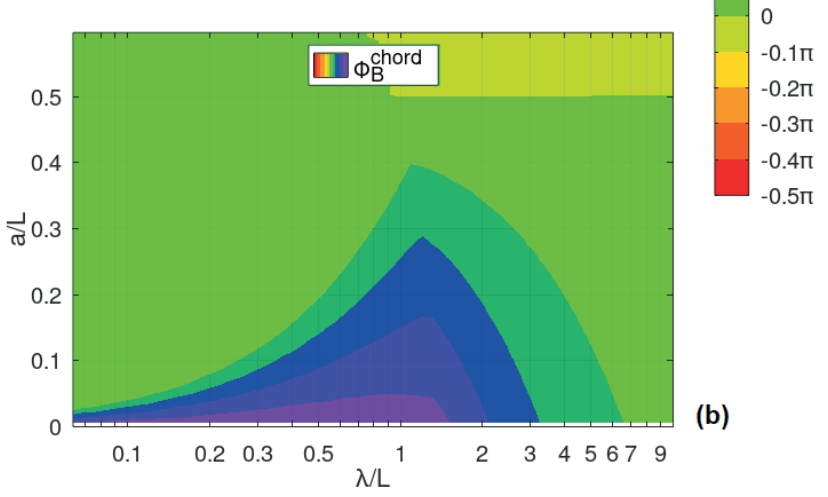

Fig. 14 Phase shift factor of shapes A (a) and B (b) due to chord measurement with wavelength $\lambda$, chord length $L$ and chord part length $a$

Summarized in formulae:

$\Phi_{C}^{\text {chord }} \equiv \Phi_{A, B, C}^{D 1} \equiv \Phi_{A, B, C}^{D 2} \equiv 0$.

\subsection{Distortion effect of filtering on hypothetical additional force maximum}

Substituting Eq. (1) into Eq. (36), for shape A yields

$F_{z, A}(x)=-\mathrm{m} \frac{4 \pi^{2}}{\lambda^{2}} v_{x}^{2} \alpha \sin \left(\frac{2 \pi}{\lambda} x\right)$.

In the same way, based on Eq. (2) for shape B yields

$F_{z, B}(x)= \begin{cases}-m \frac{4 \pi^{2}}{\lambda^{2}} v_{x}^{2} \alpha \cos \left(\frac{2 \pi}{\lambda} x\right), & 0 \leq x \leq \lambda \\ 0, & x<0, \lambda<x\end{cases}$

For shape C, vertical speed of wheel at $x=\lambda / 4$ (at the deepest point of dipped joint) is determined by the first derivative of Eq. (3) which yields

$v_{z, C}\left(\frac{\lambda}{4}\right)=\frac{\mathrm{d} p_{C}\left(\frac{\lambda}{4}\right)}{\mathrm{d} x} v_{x}=\frac{2 \pi v_{x} \alpha}{\lambda} \sin \left[\frac{2 \pi}{\epsilon \lambda}\left(\frac{\lambda}{4}-\frac{\lambda}{4}\right)\right]=0$,

as expected. Force maximum at dipped joint is

$F_{z, C}\left(\frac{\lambda}{4}\right)=\mathrm{m} \frac{\mathrm{d}^{2} p_{C}\left(\frac{\lambda}{4}\right)}{\mathrm{d} x^{2}} v_{x}^{2}=\mathrm{m} \frac{4 \pi^{2} v_{x}^{2} \alpha}{\epsilon \lambda^{2}} \cos \left[\frac{2 \pi}{\epsilon \lambda}\left(\frac{\lambda}{4}-\frac{\lambda}{4}\right)\right]$, 
thus, theoretical vertical force at elbow point is very large because

$\lim _{\epsilon \rightarrow 0}\left[F_{z, C}\left(\frac{\lambda}{4}\right)\right]=\lim _{\epsilon \rightarrow 0}\left(\frac{1}{\epsilon}\right)=\infty$.

Maximum forces for reference shape $\mathrm{A}\left(F_{z, A, \max }\right)$ and for reference shape $\mathrm{B}\left(F_{z, B, \max }\right)$ were calculated according to Eq. (56) and Eq. (57), respectively. However, in order to comparability, maximum wheel-rail forces for reference shape $\mathrm{C}\left(F_{z, C, \max }\right)$ were calculated based on the original track shapes using Eq. (36) but discretized by a $0.25 \mathrm{~m}$ step according to Eq. (37):

$$
F_{z, \max }=\mathrm{m} v_{x}^{2} \max \left(p^{\prime \prime}\right) .
$$

For showing the distortion rate of D1, D2 filters regarding this feature, values of $F_{z, \max }^{D 1}, F_{z, \max }^{D 2}$ were calculated based on filtered shapes of the reference defects illustrated via Fig. 10 with the help of the discretization according to Eq. (37):

$$
\begin{aligned}
& F_{z, \max }^{D 1}=\mathrm{m} v_{x}^{2} \max \left[\left(p^{D 1}\right)^{\prime \prime}\right], \\
& F_{z, \max }^{D 2}=\mathrm{m} v_{x}^{2} \max \left[\left(p^{D 2}\right)^{\prime \prime}\right] .
\end{aligned}
$$

In order to highlight the effect of distortion of the filtering on this feature as a function of the wavelength $(\lambda)$, the values of 1.0, 1.0, 1.0 were considered for reference shape amplitude $(\alpha)$, wheel mass $(m)$ and horizontal travelling speed $\left(v_{x}\right)$, respectively. The related results are shown in Fig. 15.

\subsection{Distortion effect of filtering on speed of hypothetical wheel lift-off}

By calculating the second-order derivatives of Eqs. (1)-(3) it can be found that for all three shapes A, B, C the unacceptable travelling speed range $v_{x}$ from the point of view of lift-off according to Eq. (39) can be theoretically defined as

$v_{x} \geq v_{x, l i f t, A}=v_{x, l i f t, B}=v_{x, l i f t, C}=\frac{\sqrt{\mathrm{g}}}{2 \pi} \frac{\lambda}{\sqrt{\alpha}}$.

The calculated maximum lift off speeds for reference shape $v_{x, l i f t, B}$ were calculated based on Eq. (64). For visualizing the distortion rate of D1, D2 filters regarding this feature, values of $v_{x, l i f t}^{D 1}, v_{x, l i f t}^{D 2}$ were calculated using Eq. (39) based on filtered shape of the reference defect B illustrated via Fig. 10, with the help of a discretization of filtered shape according to Eq. (37):
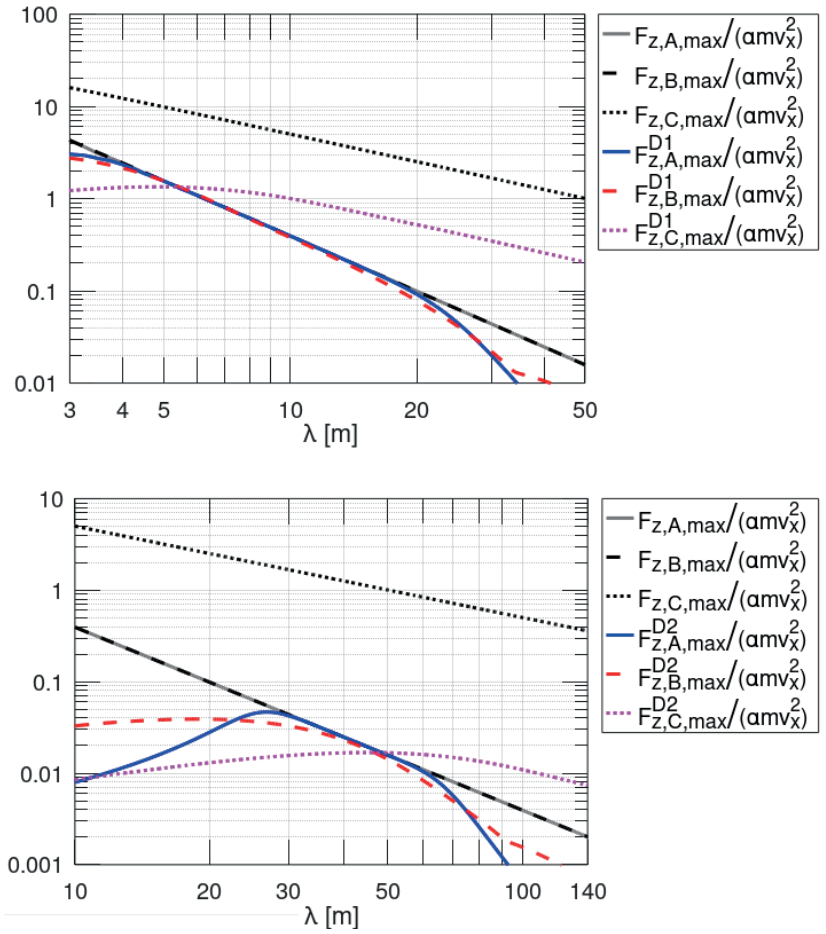

Fig. 15 Relative hypothetical force maximum in the case of passing the original $\left(F_{z, \text { max }}\right)$ and the filtered $\left(F_{z, \text { max }}^{D 1}, F_{z, \max }^{D 2}\right)$ reference shapes A, B, C

$v_{x, l i f t, B}^{D 1}=\sqrt{\frac{\mathrm{g}}{\min \left[\left(p_{B}^{D 1}\right)^{\prime \prime}\right]}}$,

$v_{x, l i f t, B}^{D 2}=\sqrt{\frac{\mathrm{g}}{\min \left[\left(p_{B}^{D 2}\right)^{\prime \prime}\right]}}$.

These critical speeds as a function of reference shape wavelength $(\lambda)$ and reference shape amplitude $(\alpha)$ were plotted in Fig. 16.

\subsection{Distortion effect of filtering on hypothetical deterioration impulse}

In this subsection, deterioration impulse is always considered between the maximum and the minimum value of $v_{z}$. Thus, impulse is calculated only for one coherent rising period of the vertical wheel momentum. Therefore, the impulses for each reference shapes based on Eqs. (40)-(43) yield:

$$
\begin{aligned}
& J_{A}=\mathrm{m} v_{z, A}(x=\lambda)-\mathrm{m} v_{z, A}\left(x=\frac{\lambda}{2}\right)=\frac{4 \pi \mathrm{m} \alpha v_{x}}{\lambda}, \\
& J_{B}=\mathrm{m} v_{z, B}\left(x=\frac{3 \lambda}{4}\right)-\mathrm{m} v_{z, B}\left(x=\frac{\lambda}{4}\right)=\frac{4 \pi \mathrm{m} \alpha v_{x}}{\lambda},
\end{aligned}
$$



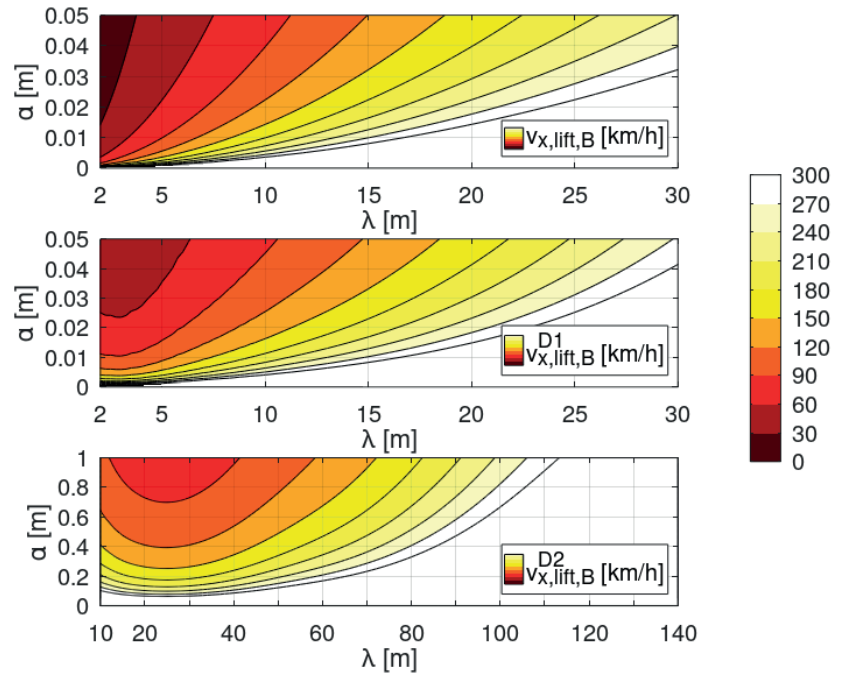

Fig. 16 Hypothetical lift off speeds passing the original $\left(v_{x, l i f f}\right)$ and the filtered $\left(v_{x, l i f t}^{D 1}, v_{x, l i f t}^{D 2}\right)$ reference shape B with wavelength $\lambda$ and with original amplitude $\alpha$

$J_{C}=\mathrm{m} v_{z, C}\left(x=\frac{\lambda}{4}+\frac{\epsilon \lambda}{4}\right)-\mathrm{m} v_{z, C}\left(x=\frac{\lambda}{4}-\frac{\epsilon \lambda}{4}\right)=\frac{4 \pi \mathrm{m} \alpha v_{x}}{\lambda}$.

For visualizing the distortion rate of D1, D2 filters regarding this feature, values of $J^{D 1}, J^{D 2}$ were calculated according to Eq. (40) based on filtered shapes of the reference defects illustrated via Fig. 10 with the help of the discretization according to Eq. (44):

$J^{D 1}=\operatorname{m} v_{x}\left\{\max \left[\left(p^{D 1}\right)^{\prime}\right]-\min \left[\left(p^{D 1}\right)^{\prime}\right]\right\}$,

$J^{D 2}=\operatorname{m} v_{x}\left\{\max \left[\left(p^{D 2}\right)^{\prime}\right]-\min \left[\left(p^{D 2}\right)^{\prime}\right]\right\}$.

In order to highlight the effect of distortion of the filtering on this feature as a function of the wavelength $(\lambda)$, the values of 1.0, 1.0, 1.0 were considered for reference shape amplitude $(\alpha)$, wheel mass $(m)$ and horizontal travelling speed $\left(v_{x}\right)$, respectively. The related results are shown in Fig. 17.

\subsection{Distortion effect of filtering on hypothetical deterioration energy}

In this subsection, deterioration energy change is always considered between the maximum and the minimum value of . Thus, according to Eq. (50) and considering Eqs. (41)-(43),

$W_{A}=\frac{\mathrm{m}}{2}\left[v_{z, A}^{2}(x=\lambda)-v_{z, A}^{2}\left(x=\frac{3 \lambda}{4}\right)\right]=\frac{2 \pi^{2} \mathrm{~m} \alpha^{2} v_{x}^{2}}{\lambda^{2}}$,
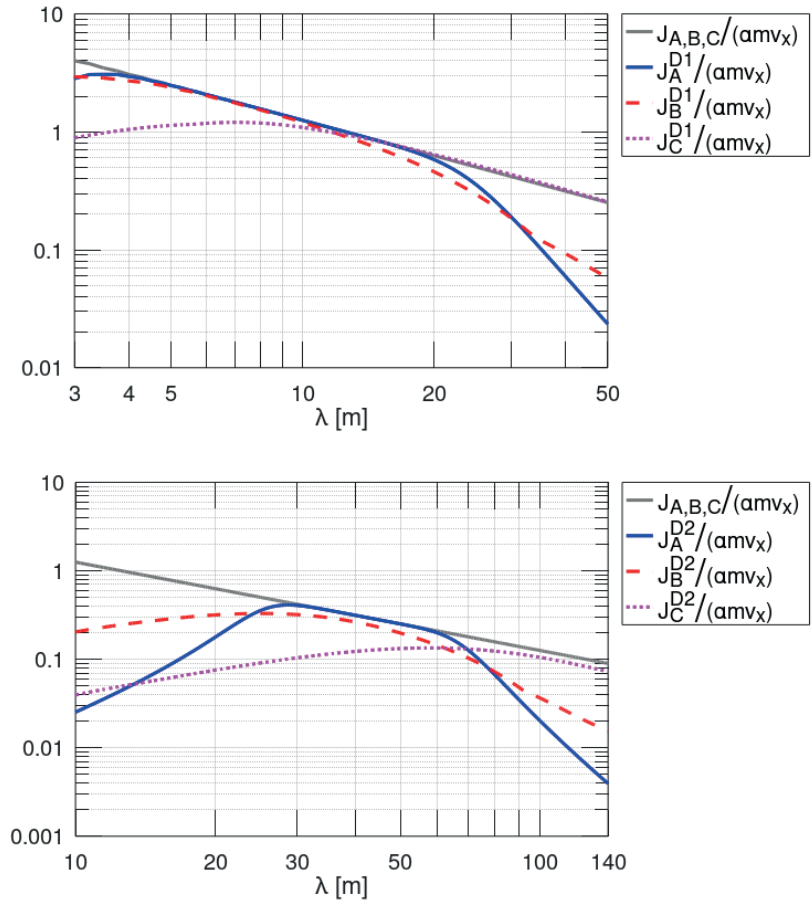

Fig. 17 Relative hypothetical deterioration impulse in the case of passing the original $(J)$ and the filtered $\left(J^{D 1}, J^{D 2}\right)$ reference shapes A, B, C

$W_{B}=\frac{\mathrm{m}}{2}\left[v_{z, B}^{2}\left(x=\frac{3 \lambda}{4}\right)-v_{z, B}^{2}\left(x=\frac{\lambda}{2}\right)\right]=\frac{2 \pi^{2} \mathrm{~m} \alpha^{2} v_{x}^{2}}{\lambda^{2}}$,

$W_{C}=\frac{\mathrm{m}}{2}\left[v_{z, C}^{2}\left(x=\frac{\lambda}{4}+\frac{\epsilon \lambda}{4}\right)-v_{z, C}^{2}\left(x=\frac{\lambda}{4}\right)\right]=\frac{2 \pi^{2} \mathrm{~m} \alpha^{2} v_{x}^{2}}{\lambda^{2}}$.

For visualizing the distortion rate of D1 and D2 filters regarding this feature, values of $W^{D 1}, W^{D 2}$ were calculated using of Eq. (49) based on filtered shapes of the reference defects illustrated via Fig. 10 with the help of the discretization according to Eq. (44):

$$
\begin{aligned}
& W^{D 1}=\frac{\mathrm{m} v_{x}^{2}}{2}\left(\max \left\{\left[\left(p^{D 1}\right)^{\prime}\right]^{2}\right\}-\min \left\{\left[\left(p^{D 1}\right)^{\prime}\right]^{2}\right\}\right), \\
& W^{D 2}=\frac{\mathrm{m} v_{x}^{2}}{2}\left(\max \left\{\left[\left(p^{D 2}\right)^{\prime}\right]^{2}\right\}-\min \left\{\left[\left(p^{D 2}\right)^{\prime}\right]^{2}\right\}\right) .
\end{aligned}
$$

In order to highlight the effect of distortion of the filtering on this feature as a function of the wavelength $(\lambda)$, the values of 1.0, 1.0, 1.0 were considered for reference shape amplitude $(\alpha)$, wheel mass $(m)$ and horizontal travelling speed $\left(v_{x}\right)$, respectively. The related results are shown in Fig. 18. 

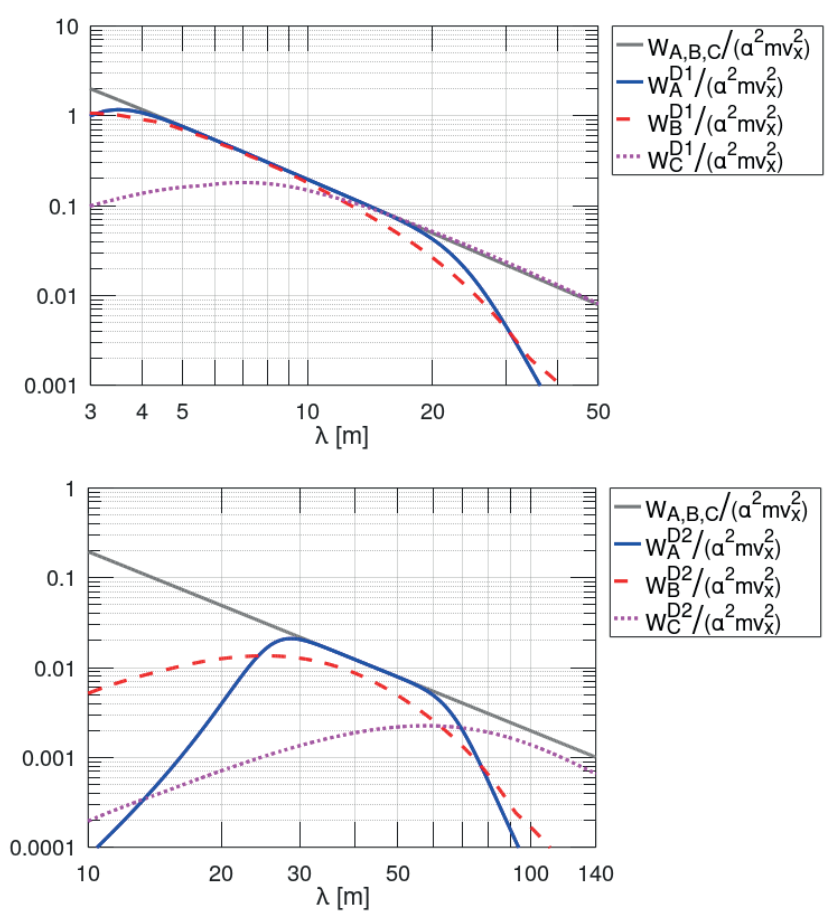

Fig. 18 Relative hypothetical deterioration energy in the case of passing the original $(W)$ and the filtered $\left(W^{D 1}, W^{D 2}\right)$ reference shapes $\mathrm{A}, \mathrm{B}, \mathrm{C}$

\section{Conclusions}

This contribution proved by analytical calculations and numerical simulations that the distortion in pattern and amplitude of generally used longitudinal level and alignment recordings are radically different depending on whether these consist of repetitive track deformations or an isolated defect.

Distortion side-effects of two different mathematical methods were investigated: versine measurement and band-pass filtering according to European regulation.

Regarding amplitude and phase distortion of versine measurement of reference shapes, diagrams were generated covering all chord lengths, chord divisions and wavelengths, comprehensively. These may play a role by planning of measurement systems. A general rule was found for zero points in case of repetitive defects: they occur where lengths of both chord parts are positive integral multiples of the track wavelength. For isolated defects, no zero points were found. This means in the practice that all kinds of isolated defects can be detected by all versine measurement systems.

As a new methodology, functions were presented which describe the distorted shape of an isolated 'cosine' track deformation measured by any versine system. It was found that the base-to-peak evaluation of an isolated defect is free of distortion if the length of the shape is not longer than twice of the shorter chord part, furthermore, in all other cases the amplitude is always reduced, as comprehensive amplitude diagrams showed.

Amplitude distortion was simulated for filtered signals according to the standard EN 13848-1. Amplitude reduction functions were plotted considering D1 and D2 filters. It was found that base-to-peak evaluation method of filtered signals of 'isolated cosine' shape show a significant amplitude reduction comparing to the real shapes, even at wavelengths which correspond to the transition band of the filter. It means in the practice, that measurement diagram of recording car shows always smaller base-topeak value than the real amplitude of the isolated 'cosine' deflection. Therefore, further vehicle-track simulations based on D1 values can be problematic. Experts may consider the possibility of broader use of peak-to-peak evaluation which approximates the real amplitude more closely.

Derivatives of the filtered signals were also investigated in the light of the newly proposed force, acceleration, impulse, and energy features. Analytical considerations showed that the instantaneous additional vertical force passing an elbow point (reference shape C) tends to be infinite, however, the dissipated impulse and energy are finite, and their formulae were given. Nevertheless, dipped joint is one of the central phenomena of track degradation because the track experiences a concentrated energy quantum here.

The theoretical speed of hypothetical wheel lift-off, which may be considered by experts while defining of track geometry tolerance values, showed unexpectedly low values in the case of short wavelengths. It should be noted, that these results are largely theoretical because the used model is simply: it assumes an independent wheel and rigid track.

Simulations showed that changes due to the 'D1 filtering' of 'isolated cosine' shape in the 'force features' as well as in hypothetical 'deterioration impulse' and 'deterioration energy' are not significant. It means that standardized filtering method (according to European standard in force) preserves the derivatives of the isolated track defect shapes much better than the amplitude of them.

Methodology of this contribution can be applied to road surface irregularities or other surface evenness measurements as well. 


\section{References}

[1] EUR-Lex \& Legal Information Unit "Commission Regulation (EU) No 1299/2014 of 18 November 2014 on the technical specifications for interoperability relating to the 'infrastructure' subsystem of the rail system in the European Union", Official Journal of the European Union, Luxembourg, 2014. [online] Available at: http://data.europa. eu/eli/reg/2014/1299/oj

[2] Weston, P., Roberts, C., Yeo, G., Stewart, E. "Perspectives on railway track geometry condition monitoring from in-service railway vehicles", Vehicle System Dynamics, 53(7), pp. 1063-1091, 2015. https://doi.org/10.1080/00423114.2015.1034730

[3] Chiou, S.-B., Yen, J.-Y. "Precise railway alignment measurements of the horizontal circular curves and the vertical parabolic curves using the chord method", Proceedings of the Institution of Mechanical Engineers, Part F: Journal of Rail and Rapid Transit, 233(5), pp. 537-549, 2019.

https://doi.org/10.1177/0954409718800527

[4] Lichtberger, B. "Track maintenance", In: Track compendium, Eurailpress Tetzlaff-Hestra GmbH \& Co. KG, Hamburg, Germany, 2005, pp. 417-418.

[5] Mauer, L. "Determination of track irregularities and stiffness parameters with inverse transfer functions of track recording vehicles", Vehicle System Dynamics, 24(sup1), pp. 117-132, 1995. https://doi.org/10.1080/00423119508969619

[6] Wang, P., Wang, Y., Tang, H., Gao, M., Chen, R., Xu, J. "Error theory of chord-based measurement system regarding track geometry and improvement by high frequency sampling", Measurement, 115, pp. 204-216, 2018.

https://doi.org/10.1016/j.measurement.2017.10.019

[7] Insa, R., Inarejos, J., Salvador, P., Baeza, L. "On the filtering effects of the chord offset method for monitoring track geometry", Proceedings of the Institution of Mechanical Engineers, Part F: Journal of Rail and Rapid Transit, 226(6), pp. 650-654, 2012. https://doi.org/10.1177/0954409712447481

[8] CEN "EN 13848-1:2019 Railway applications - Track - Track geometry quality - Part 1: Characterization of track geometry", European Committee for Standardization, Brussels, Belgium, 2019.

[9] Haigermoser, A., Luber, B., Rauh, J., Gräfe, G. "Road and track irregularities: measurement, assessment and simulation", Vehicle System Dynamics, 53(7), pp. 878-957, 2015. https://doi.org/10.1080/00423114.2015.1037312
[10] Luber, B., Haigermoser, A., Grabner, G. "Track geometry evaluation method based on vehicle response prediction", Vehicle System Dynamics, 48(S1), pp. 157-173, 2010. https://doi.org/10.1080/00423111003692914

[11] Sowiński, B. "Interrelation between wavelengths of track geometry irregularities and rail vehicle dynamic properties", Archives of Transport, 25, pp. 97-108, 2013.

[12] Wu, H., Wilson, N. "Railway vehicle derailment and prevention", In: Iwnicki, S. (ed.) Handbook of railway vehicle dynamics, CRC Press, Boca Raton, USA, 2006, pp. 209-237. https://doi.org/10.1201/9780849333217.ch8

[13] Karis, T., Berg, M., Stichel, S., Li, M., Thomas, D., Dirks, B. "Correlation of track irregularities and vehicle responses based on measured data", Vehicle System Dynamics, 56(6), pp. 967-981, 2018. https://doi.org/10.1080/00423114.2017.1403634

[14] Soleimanmeigouni, I., Ahmadi, A., Nissen, A., Xiao, X. "Prediction of railway track geometry defects: a case study", Structure and Infrastructure Engineering, 16(7), pp. 987-1001, 2020. https://doi.org/10.1080/15732479.2019.1679193

[15] Li, M., Persson, I., Spännar, J., Berg, M. "On the use of second-order derivatives of track irregularity for assessing vertical track geometry quality", Vehicle System Dynamics, 50(sup1), pp. 389-401, 2012. https://doi.org/10.1080/00423114.2012.671947

[16] Ágh, C. "Comparative Analysis of Axlebox Accelerations in Correlation with Track Geometry Irregularities", Acta Technica Jaurinensis, 12(2), pp. 161-177, 2019. https://doi.org/10.14513/actatechjaur.v12.n2.501

[17] Andor, K., Polgár, R. "Localization of bearing errors using spline method", Periodica Polytechnica Civil Engineering, 58(4), pp. 339345, 2014. https://doi.org/10.3311/PPci.7528

[18] Barna, Z., Kisgyörgy, L. "Analysis of Hyperbolic Transition Curve Geometry", Periodica Polytechnica Civil Engineering, 59(2), pp. 173-178, 2015. https://doi.org/10.3311/PPci.7834

[19] Farkas, A. "Measurement of Railway Track Geometry: A State-ofthe-Art Review", Periodica Polytechnica Transportation Engineering, 48(1), pp. 76-88, 2020. https://doi.org/10.3311/pptr.14145

[20] Smith, S. W. "Convolution", In: Digital Signal Processing, Newnes, Burlington, MA, USA, 1997, pp. 107-122. https://doi.org/10.1016/b978-0-7506-7444-7/50043-1 